\title{
De l'autre côté du miroir
}

\author{
par Douglas Doucette
}

Alors que nous entamons les derniers mois de l'année 2020, je suppose que bon nombre d'entre nous ont souvent eu l'impression de traverser le miroir, comme dans le roman du XIX ${ }^{\mathrm{e}}$ siècle de Lewis Carroll, qui raconte des voyages fantastiques et décrit des interactions avec des personnages invraisemblables et inattendus. La plupart des Canadiens n'ont jamais connu de pandémie et ne savaient vraisemblablement pas à quoi s'attendre lorsque les comptes-rendus sur la COVID-19 et sa propagation imminente à l'échelle planétaire ont commencé à circuler.

Notre vie professionnelle et personnelle a été irrémédiablement bouleversée. La pandémie a obligé les associations sans but lucratif, comme la Société canadienne des pharmaciens d'hôpitaux (SCPH), à adapter leurs activités de bureau ainsi que les programmes et les services qu'elles offraient à leurs membres. Le Conseil et les succursales de la $\mathrm{SCPH}$ ont réexaminé leurs priorités, mis davantage l'accent sur certaines activités et reporté ou suspendu d'autres à cause des pressions financières exacerbées par la pandémie. Le Journal canadien de la pharmacie hospitalière (JCPH) a été directement touché par des mesures de réduction des coûts. Afin de préserver la grande qualité du Journal et de diminuer les coûts de production, le nombre de numéros du JCPH a été réduit à quatre par an au lieu de six. De plus, le système de soumission du JCPH et son interface de publication sont en cours de migration vers un système plus efficace et plus économique qui permettra de rationaliser le flux de travail. Il offrira en outre aux lecteurs davantage de fonctionnalités.

Au cours de ces derniers mois, j’ai réfléchi aux expériences que j'ai vécues au cours de mon mandat de trois ans en tant que président de la SCPH. J'ai eu l'occasion de rencontrer des acteurs clés de Santé Canada, de l'ANORP, de l'ACEIP, de l'ACSP et d'autres, qui m'ont fait part de points de vue divers sur les enjeux auxquels font face les soins de santé et la pharmacie. En tant que président, j’ai répondu aux questions des médias et d'autres associations, comme on peut s'y attendre, mais leurs thèmes et leur calendrier n'étaient pas toujours prévisibles. Je remercie le personnel du bureau et les collègues de l'équipe présidentielle de leur soutien pendant que je me préparais à ces entrevues. Cependant, je n'ai pas pu anticiper certaines situations, comme le départ à la retraite de Myrella Roy, notre directrice générale de longue date, peu après mon élection à la présidence. Ce fut un privilège de travailler avec elle pendant une partie de mon mandat, mais j’ai dû ensuite diriger le groupe de travail chargé de trouver son successeur. En 2019, le Conseil a vécu des moments passionnants lors du choix de la nouvelle directrice générale, Jody Ciufo, et quant à moi, ce fut un privilège de l'accompagner dans sa prise de contact avec la SCPH et la pharmacie hospitalière en général. Nous avons travaillé main dans la main sur la Stratégie de développement durable pour établir notre feuille de route future en tant quassociation nationale. Nous avons également pris le temps de célébrer le $50^{\mathrm{e}}$ anniversaire de notre Congrès sur la pratique professionnelle et de réfléchir sur le long chemin parcouru par notre profession, en partie grâce au leadership de la SCPH et de ses membres.

Le Plan stratégique de 2020-2023 de la SCPH sera bientôt rendu public (si ce n'est pas déjà fait). Il trace la voie à suivre pour améliorer la valeur de la Société pour ses membres ainsi que sa stabilité fiscale au moyen de priorités stratégiques bien définies. En tant que porte-parole de la pharmacie hospitalière au Canada, la SCPH a continué de faire preuve d'un solide leadership à l'égard d'enjeux clés pour la défense des intérêts dans les domaines du Programme national d'assurance-médicaments, de la déclaration obligatoire de réactions adverses aux médicaments et de l'importation aux É.-U. de médicaments canadiens. À la fin de 2020, les recommandations émanant de notre groupe de travail sur le cannabis et de celui sur les techniciens en pharmacie devraient être soumises à l'examen du Conseil pour qu'il oriente les travaux dans ces deux domaines.

Il y a trois ans, peu d'entre nous auraient pu imaginer où nous en serions en tant que Société aujourd'hui. Si nous regardons de l'autre côté du miroir et au-delà, il est peutêtre encore plus difficile de prédire l'avenir à la lumière de la pandémie de COVID-19 et de l'envergure de ses effets. L'engagement et le professionnalisme que démontrent les membres des équipes de la pharmacie hospitalière tant pour conduire la riposte que pour gérer les pénuries de médicaments et les soins aux patients en ces moments difficiles illustrent combien notre profession est essentielle à la santé des Canadiens. Je suis heureux d'avoir été au service de cette société nationale solide, résolue et engagée en tant que membre de l'équipe présidentielle, et je me réjouis de voir ce que nous accomplirons au cours des années à venir.

[Traduction par l'éditeur]

Douglas Doucette, B. Sc. (Pharm.), Pharm. D., F.C.S.H.P., était au moment de la rédaction, président sortant et agent de liaison externe pour la Société canadienne des pharmaciens d'hôpitaux (SCPH). En date du 17 octobre 2020, il a terminé son mandat de trois ans en tant que membre de l'équipe présidentielle de la SCPH. 